\title{
La dynamique spatio-temporelle des brouillards de rayonnement dans le fossé rhénan méridional à l'aide d'images NOAA-AVHRR
}

\section{Laurent Wahl}

\section{(2) OpenEdition \\ Journals}

Édition électronique

URL : https://journals.openedition.org/rge/4251

DOI : 10.4000/rge.4251

ISSN : 2108-6478

Éditeur

Association des géographes de l'Est

Édition imprimée

Date de publication : 1 janvier 2000

ISSN : 0035-3213

Référence électronique

Laurent Wahl, «La dynamique spatio-temporelle des brouillards de rayonnement dans le fossé rhénan méridional à l'aide d'images NOAA-AVHRR », Revue Géographique de l'Est [En ligne], vol. 40 / 1-2 | 2000, mis en ligne le 07 août 2013, consulté le 28 juin 2022. URL : http://journals.openedition.org/rge/4251 ; DOI : https://doi.org/10.4000/rge.4251

Ce document a été généré automatiquement le 29 septembre 2020

Tous droits réservés 


\title{
La dynamique spatio-temporelle des brouillards de rayonnement dans le fossé rhénan méridional à l'aide d'images NOAA-AVHRR
}

\author{
Laurent Wahl
}

Thèse soutenue à l'Université Louis-Pasteur de Strasbourg le 10 janvier 1997 sous la direction du professeur Patrice Paul

\section{Problématique}

1 Cette étude s'insère dans le cadre du programme de recherche transfrontalier REKLIP (REgio-KLIma-Projekt) dont l'objectif est d'étudier d'une manière approfondie le climat du Fossé Rhénan méridional.

2 Par sa configuration topographique, le Fossé Rhénan méridional est souvent affecté par des brouillards de rayonnement qui apparaissent principalement en automne et en hiver. Ces brouillards peuvent persister pendant plusieurs jours et occasionner de nombreuses contraintes au niveau du trafic routier et aérien. Ils contribuent également à une détérioration de la qualité de l'air compte tenu de l'accumulation des polluants qu'ils induisent. C'est pourquoi une étude sur la dynamique spatio-temporelle des brouillards s'avère nécessaire dans un espace aussi densément peuplé et relativement industrialisé.

3 Le brouillard est un phénomène météorologique qui présente une extension à la fois horizontale et verticale, fort variable dans le temps et dans l'espace. Les observations effectuées dans les stations synoptiques ne suffisent pas à donner une idée précise de l'extension de ce phénomène et de sa dynamique étant donné leur éloignement respectif. Seule la combinaison des données climatologiques traditionnelles avec l'imagerie satellitale permet de mieux cerner l'extension spatio-temporelle des brouillards. 


\section{Objectifs}

Ce travail a été réalisé avec un échantillon de scènes NOAA-AVHRR ${ }^{1}$ suffisamment représentatif. Les informations fournies par les données NOAA-AVHRR sont combinées avec des données climatiques, afin de mieux comprendre les facteurs physiques et climatiques qui peuvent conduire vers telle ou telle extension du brouillard. Les objectifs étaient les suivants :

5 a) La cartographie de l'extension des brouillards persistants (Fig. 1) sous la forme de cartes de fréquences d'apparition selon la direction et l'intensité du flux synoptique observé vers 1500 mètres d'altitude (niveau de pression atmosphérique 850 hectopascals).

Fig. 1 : Extensions du brouillard observées selon un flux synoptique donné vers $850 \mathrm{hPa}$
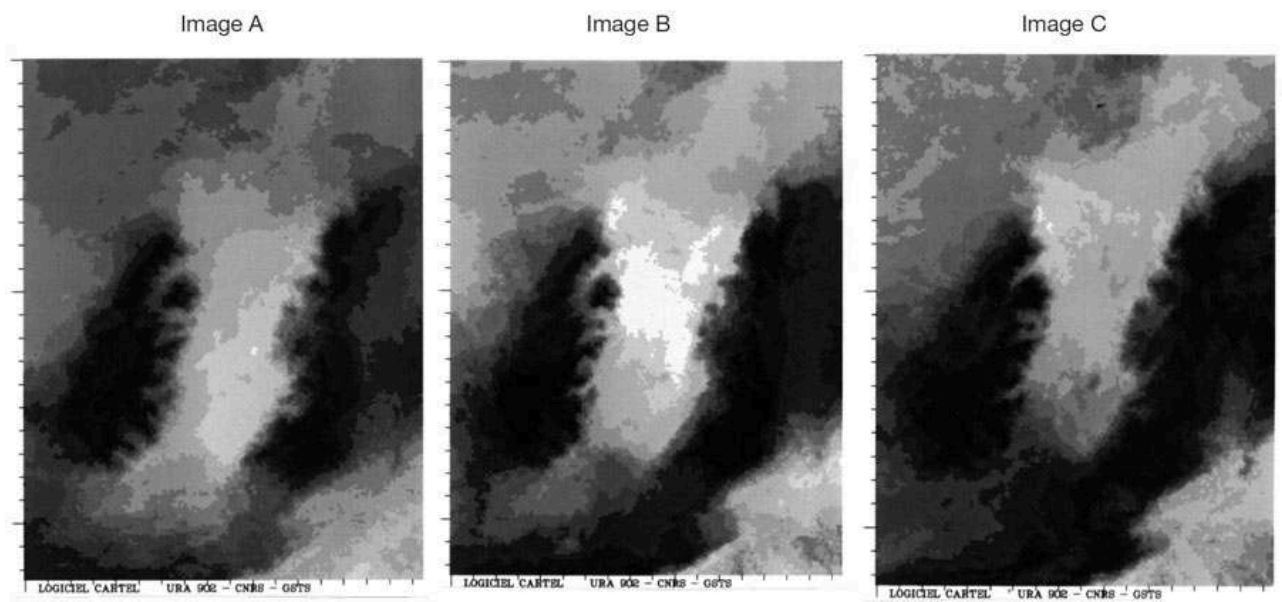

NB : Les fréquences d'occurrences s'échelonnent entre $0 \%$ (couleur noire) et $90 \%$ (couleur blanche). A : Flux de secteur N à NE ; B : Vents variables et faibles ; C : Flux de secteur S à SW.

b) La détermination de l'altitude de la couche limite supérieure du brouillard à partir de sommets repères émergeant de la nappe et d'un Modèle Numérique de Terrain (MNT).

c) Un essai de classification des champs d'extension du brouillard suivant leur forme géométrique (classification empirique) et selon un algorithme de classification hiérarchique ascendante à partir des variables climatiques.

d) Une analyse de l'évolution des facteurs climatiques qui interviennent au cours des différentes phases de formation et de dissipation d'un brouillard de rayonnement.

Le but recherché est d'apporter un outil complémentaire et opérationnel à la prévision de l'extension des brouillards. Les résultats obtenus dans cette étude devraient aider les responsables dans le domaine de l'aménagement et des transports à prendre des décisions adéquates vis-à-vis de ce type de phénomène climatique.

\section{Méthodologie détaillée}

10 a) La cartographie de l'extension des brouillards a été réalisée à partir d'un échantillon de 96 scènes sélectionnées durant le semestre froid (octobre à mars) sur une durée de 11 ans (1982-1993). La résolution spatiale des images $(1,1 \mathrm{~km})$ s'avère suffisante pour mettre en évidence des variations locales significatives. Les images NOAA-AVHRR 
utilisées pour la réalisation des cartes ne concernent que les orbites du début de l'après-midi. Par conséquent, il s'agit d'une cartographie des brouillards persistants. Chaque image a été corrigée géométriquement par la méthode des points amers ${ }^{2}$ afin de pouvoir superposer l'ensemble des scènes. L'extension du brouillard, sur chaque scène, a été discriminée à partir d'une méthode de classification par hyperboittes ${ }^{3}$ qui combine l'information fournie par les divers canaux en créant une image de synthèse en noir et blanc, appelée dichotomie. Cette image est codée en langage binaire $0 / 1$ (présence - absence de brouillard). L'ensemble des scènes dichotomisées a été superposé afin d'obtenir une image de synthèse montrant l'extension spatiale du brouillard selon des fréquences d'occurrence pour chaque secteur donné du Fossé Rhénan.

11 b) Selon les informations provenant des stations de radiosondages de Nancy et de Payerne ${ }^{4}$ ainsi que de la station du Feldberg (1 $493 \mathrm{~m}$ ), les images ont pu être classées et superposées en fonction des conditions synoptiques de vent et de pression observées à $850 \mathrm{hPa}$. Sur chaque image dichotomisée, les contours du brouillard ont été individualisés et superposés à un MNT, afin d'obtenir une altitude moyenne des contours.

12 c) Une classification des champs d'extension du brouillard a été réalisée en utilisant deux types de méthodes :

13 - Une méthode empirique, basée sur le regroupement des formes d'extension similaires de la nappe afin de vérifier si l'on retrouve des paramètres climatiques identiques pour chaque classe.

14 - Une méthode statistique, basée sur l'utilisation d'un algorithme de classification hiérarchique ascendante à partir des variables climatiques représentatives d'une extension donnée $\mathrm{du}$ brouillard. L'algorithme regroupe les variables en sousensembles, les plus homogènes possibles, à partir des dissemblances qui peuvent exister entre les différents individus. L'algorithme va regrouper en classes les éléments qui présentent les valeurs de dissemblance les plus faibles.

15 d) À partir de données climatiques issues de 4 pylônes de mesure, d'une hauteur de 120 à 200 mètres, situés le long du Rhin en territoire allemand, les processus climatiques survenant dans les basses couches atmosphériques, au cours de la formation et de la dissipation du brouillard, ont pu être étudiés et individualisés sous la forme de phases.

\section{Résultats}

16 a) La cartographie des fréquences d'occurrence des brouillards a permis de délimiter, d'une manière objective, les secteurs où le brouillard a tendance à persister selon des conditions de vent et de gradient de pression donnés (Fig. 1).

17 Ces secteurs se localisent essentiellement dans la partie centrale du Fossé Rhénan, là où l'effet d'abri exercé par le relief est le plus marqué, notamment entre Strasbourg et Colmar. Cette cartographie a permis également de visualiser les secteurs où la nappe a le plus de chance de se dissiper en cours de journée. Ils se situent au niveau des trouées (Belfort, Kraichgau) où les conditions de ventilation sont meilleures. Cette étude a permis de démontrer que la localisation de ces secteurs varie géographiquement selon l'orientation du flux dominant en altitude. L'effet d'abri, face au flux dominant, change géographiquement d'emplacement : par flux orienté au secteur NE à E, c'est la partie 
méridionale du Pays de Bade qui est la plus affectée, tandis que par flux de secteur S à SW, c'est le secteur situé le long du piémont vosgien.

b) La détermination de l'altitude supérieure du brouillard a permis d'identifier deux limites altitudinales de stagnation préférentielles : l'une située entre 400 et 500 mètres et l'autre vers 600 mètres. La première correspond à la limite supérieure du brouillard (brouillard dense jusqu'en plaine) et la seconde à celle des stratus bas. La comparaison des résultats issus des 2 méthodes (MNT et sommets repères) avec des données vérifiées sur le terrain a permis d'établir que MNT a tendance à sous-estimer l'altitude du toit de la nappe.

c) La classification des champs de brouillard a permis de démontrer l'existence d'une relation entre la répartition spatiale observée et les conditions synoptiques. À chaque forme d'extension analogue de la nappe correspond une situation climatologique caractéristique (Fig. 2 et tableau 1).

Fig. 2 : Principales extensions du brouillard observées en début d'après-midi dans le Fossé Rhénan méridional (le brouillard est ici en noir)

\begin{tabular}{|c|c|c|c|c|c|c|c|c|}
\hline 1 & 2 & 3 & 4 & 5 & 6 & 7 & 8 & 9 \\
\hline & & & & & \\
\hline
\end{tabular}

Tableau 1 : Influence de la variation du flux présent au Feldberg sur le type de configuration spatiale observé et l'évolution de la couche-limite supérieure du brouillard en relation avec le gradient de pression

\begin{tabular}{|c|c|c|c|c|}
\hline $\begin{array}{c}\text { Évolution } \\
\text { du flux } \\
\text { synoptique } \\
\text { vers } 850 \mathrm{hPa}\end{array}$ & $\begin{array}{l}\text { Gradient } \\
\text { de pression } \\
\text { Payerne- } \\
\text { Strasbourg }\end{array}$ & $\begin{array}{l}\text { Évolution } \\
\text { de la situation } \\
\text { synoptique }\end{array}$ & $\begin{array}{l}\text { Évolution } \\
\text { de la limite } \\
\text { supérieure } \\
\text { des brouillards }\end{array}$ & $\begin{array}{c}\text { Type de } \\
\text { configuration } \\
\text { spatiale } \\
\text { du brouillard }\end{array}$ \\
\hline $\begin{array}{c}\text { Maintien } \\
\text { du flux d'E à NE }\end{array}$ & $\begin{array}{c}\text { Négatif } \\
\text { en s'atténuant } \\
\text { de }-1,0 \text { à }-0,2 \mathrm{hPa}\end{array}$ & AC dominant & $\begin{array}{l}\text { Augmentation } \\
\text { moyenne de } 50 \mathrm{~m}\end{array}$ & $\begin{array}{l}\text { Extension } \\
\text { de type } 1 \text { dominante }\end{array}$ \\
\hline $\begin{array}{c}\text { Changement } \\
\text { d'orientation } \\
\text { au secteur S à SW }\end{array}$ & $\begin{array}{c}\text { Gradient } \\
\text { devenant positif }\end{array}$ & $\begin{array}{l}\text { AC évoluant } \\
\text { vers AS }\end{array}$ & $\begin{array}{c}\text { Diminution } \\
\text { moyenne } \\
\text { de } 100 \text { à } 200 \mathrm{~m}\end{array}$ & $\begin{array}{c}1 \text { ou } 8 \text { évoluant } \\
\text { vers } 6\end{array}$ \\
\hline $\begin{array}{l}\text { Maintien d'un flux } \\
\text { de } S \text { à SW }\end{array}$ & $\begin{array}{l}\text { Gradient positif } \\
\text { qui se renforce } \\
\text { de }+1,1 \text { à }+2,0\end{array}$ & $A C$ ou $A S$ & $\begin{array}{c}\text { Diminution } \\
\text { moyenne de } 80 \mathrm{~m}\end{array}$ & $\begin{array}{l}\text { Extension } \\
\text { de type } 6 \\
\text { dominante }\end{array}$ \\
\hline $\begin{array}{l}\text { Changement } \\
\text { d'orientation } \\
\text { au secteur E à NE }\end{array}$ & $\begin{array}{c}\text { Gradient } \\
\text { devenant négatif }\end{array}$ & $\begin{array}{c}\text { Maintien } \\
\text { d'une situation } \\
\text { de type } A C\end{array}$ & $\begin{array}{l}\text { Augmentation } \\
\text { moyenne } \\
\text { de } 100 \text { à } 200 \mathrm{~m}\end{array}$ & 5 évoluant vers 1 \\
\hline
\end{tabular}

AC : Anticyclone centré sur l'Europe centrale avec un flux d'Est à NE dans les basses couches ; AS :Anticyclone centré sur l'arc alpin et le SE de l'Europe générant un flux de S à SW en altitude.

L'extension 1 se présente sous la forme d'une nappe de stratus bas qui recouvre l'ensemble du fossé Rhénan à l'exception des zones de relief situées au-dessus de 800 mètres d'altitude. L'extension 2 témoigne d'un retrait des brouillards persistants dans la région des Trois-Frontières (Saint-Louis, Mulhouse-Bâle) sous l'action d'un écoulement catabatique d'air froid issu du plateau Suisse. Les situations 3, 4 et 5 montrent une 
extension réduite des brouillards dans le tiers méridional du fossé Rhénan au-delà de Colmar compte tenu d'une meilleure ventilation par flux de SW. Les extensions 6, 7 et 9 du brouillard sont réduites à des îlots localisés en bordure des massifs ou au centre du fossé, là, où l'effet d'abri face aux vents dominants est le plus marqué. L'extension 8 témoigne d'une accumulation des brouillards et stratus bas dans les 2 tiers Sud du fossé sous l'effet du vent de NE qui assure un meilleur brassage de l'air au Nord de la région strasbourgeoise.

21 d) L'utilisation de données climatiques issues de pylônes de mesures a permis de confirmer l'existence de phases climatiques analogues à celles qui avaient été identifiées dans d'autres régions (Albany, USA, en 1982 ; Valladolid, Espagne, 1985 ; Bologne, Italie, 1986). Une situation synoptique favorable à la formation du brouillard se produit lors de conditions anticycloniques avec un flux synoptique de secteur $E$ à NE à la faveur d'un refroidissement radiatif nocturne intense. L'apparition d'un brouillard dense a lieu, le plus fréquemment, en seconde partie de nuit (vers 4 ou 5 heures du matin) avec une orientation des vents au secteur $\mathrm{SW}$ avec des vitesses inférieures à $2 \mathrm{~m} / \mathrm{s}$ dans les basses-couches.

La phase de dissipation s'accompagne fréquemment d'une élévation de température et d'un accroissement plus ou moins marqué de la vitesse du vent. Elle se produit en général vers midi, moment privilégié, où l'intensité du rayonnement solaire global incident est maximale. La dissipation du brouillard est plus précoce dans la partie Sud que dans la partie Nord du Fossé Rhénan, surtout lorsqu'on est en présence d'une advection d'air doux et turbulent de secteur $\mathrm{S}$ à $\mathrm{SW}$ avant l'approche d'un système perturbé.

\section{Conclusion}

Cette étude a permis de mettre en évidence la complexité des interactions qui existent entre l'évolution des conditions synoptiques, la configuration des reliefs environnants et les conditions de formation et d'extension des brouillards d'inversion dans le Fossé Rhénan méridional. Selon l'orientation du flux synoptique vers $850 \mathrm{hPa}$, le relief crée soit un effet d'abri propice à la formation et à la stagnation du brouillard, soit un effet d'accélération des vents, favorable à sa dissipation, tributaires de l'évolution des conditions synoptiques. Le flux de secteur S à SW favorise le démantèlement progressif de la couche d'inversion par la partie méridionale du fossé rhénan (effet de la trouée de Belfort).

D'autre part, cette étude montre que certaines extensions du brouillard se reproduisent à des intervalles de temps plus ou moins réguliers selon des conditions synoptiques analogues. L'utilisation de pylônes de mesures a permis d'effectuer un suivi de l'enchaînement des divers processus physiques (processus advectifs et radiatifs) qui interviennent lors des différentes phases de formation et de dissipation. L'apparition et la succession des phases dépendent de l'interaction de ces processus qui sont souvent tributaires de l'évolution des conditions synoptiques.

Cette étude souligne la complexité des interactions qui existent entre les reliefs et les divers processus radiatifs et advectifs qui rendent difficiles les perspectives opérationnelles d'une modélisation du brouillard (effets indirects des reliefs environnants à introduire dans le modèle, problème d'initialisation fine du modèle, adaptation de la taille des mailles du modèle à l'échelle de la région...). Néanmoins les 
résultats obtenus ouvrent des perspectives pour la prévision à courte et moyenne échéance de l'extension spatio-temporelle des brouillards de rayonnement pour le Fossé Rhénan.

\section{NOTES}

1. NOAA-AVHRR : satellite héliosynchrone équipé d'un capteur AVHRR d'une résolution d'un kilomètre.

2. Amers : points de repères géographiques servant à caler les images sur un fond cartographique de référence.

3. Hyperboîte : limites radiométriques créées à partir des différents canaux d'une scène NOAAAVHRR. En croisant ces limites on obtient des intervalles qui constituent les hyperboîtes.

4. Payerne : ville située dans le canton de Fribourg en Suisse francophone.

\section{AUTEUR}

\section{LAURENT WAHL}

Université de Nancy 2 - UFR des sciences historiques, géographiques et de musicologie - 3, place Godefroi de Bouillon, B.P. 3397 - 54015 Nancy 